$12 ; 13$

\title{
Релятивистская лампа обратной волны с продольно-щелевым дифракционным выходом
}

\author{
(C) В.В. Кладухин, С.П. Храмцов, В.Ю. Ялов \\ Институт электрофизики УрО РАН, Екатеринбург, Россия \\ E-mail: laepr@iep.uran.ru
}

Поступило в Редакцию 29 сентября 2021 г.

В окончательной редакции 21 октября 2021 г.

Принято к публикации 21 октября 2021 г.

\begin{abstract}
Изложены результаты экспериментальных исследований по оценке влияния продольных щелей в коническом дифракционном выходе $10 \mathrm{GHz}$ релятивистской лампы обратной волны с рабочей модой $\mathrm{TM}_{01}$, совмещенном с коллектором электронов. Экспериментально показано, что замена сплошного конического волновода на подобный ему продольно-щелевой волновод слабо влияет на рабочие характеристики лампы. Переход от сплошного коллектора к продольно-щелевому позволяет уменьшить объем коллекторной плазмы и микрочастиц, образующихся на поверхности дифракционного выхода под действием электронного пучка, улучшить их адсорбцию и удаление.
\end{abstract}

Ключевые слова: релятивистский СВЧ-генератор, лампа обратной волны, дифракционный выход, коллектор электронов, коллекторная плазма.

DOI: 10.21883/PJTF.2022.03.51979.19039

Сильноточные релятивистские лампы обратной волны (ЛОВ) сантиметрового диапазона длин волн, использующие конусообразный адиабатический дифракционный выход, совмещенный с коллектором электронов, разрабатываются и исследуются последние несколько десятилетий [1-3]. Отмечено, что под действием падающих электронов происходит заполнение дифракционного выхода коллекторной плазмой, десорбированными газами, парами и микрочастицами материала коллектора, отрицательно влияющее на работу ЛОВ в частотных режимах, а также при формировании длинных микроволновых импульсов. Существенное изменение режимов генерации плазменно-релятивистских СВЧ-генераторов под действием плазмы, микрочастиц и ионного фона, образующихся в области коллектора под действием электронного пучка, отмечено в работе [4]. В связи с этим представляют интерес конструктивные решения, меняющие условия проявления перечисленных выше явлений, за счет перехода от сплошных волноводных систем к щелевым.

Поскольку в рассматриваемых ЛОВ используются осесимметричные волны ТМ-типа, имеется принципиальная возможность замены традиционно используемых сплошных осесимметричных волноводных систем на их продольно-щелевые аналоги.

Далее приведены результаты экспериментального исследования изменения рабочих характеристик ЛОВ при замене сплошного конического дифракционного выхода, совмещенного с коллектором электронов, на его продольно-щелевые аналоги, а также оценка прозрачности таких коллекторов для электронного пучка.

Эксперименты были выполнены на основе трехсантиметровой ЛОВ с рабочей модой $\mathrm{TM}_{01}$, аналогичной применявшейся в [5], использующей сплошную прямоугольно-гофрированную замедляющую волновую структуру (SWS) и резонансный рефлектор-модулятор (RRM), предложенный в [6]. Длина SWS равна $120 \mathrm{~mm}$, больший диаметр гофры $32 \mathrm{~mm}$, глубина гофрировки в основной части SWS $2 \mathrm{~mm}$, период $15 \mathrm{~mm}$, расстояние между SWS и RRM $\left(L_{d r}\right) 20.5 \mathrm{~mm}$. Конструктивная схема ЛОВ приведена на рис. $1, a$.

В ЛОВ использовался сильноточный тонкостенный электронный пучок (e-beam на рис. 1,a) трубчатой формы со следующими параметрами: энергия электронов $\sim 650 \mathrm{keV}$, ток пучка $\sim 8 \mathrm{kA}$, длительность импульсов ускоряющего напряжения и тока электронного пучка $\sim 15 \mathrm{~ns}$, режим работы одиночно-импульсный. Трубчатую форму пучка обеспечивали графитовый катод (эмиттер электронов - Cath) с кольцевой взрывоэмиссионной кромкой и осесимметричное направляющее магнитное поле $\left(H_{0}\right)$, создаваемое импульсным соленоидом $(\mathrm{Sol})$. Для формирования наносекундных импульсов ускоряющего напряжения использовался высоковольтный генератор на основе 25-омной формирующей линии, подключаемый к 80-омному входу ЛОВ через трансформирующую линию.

На регулярном участке замедляющей системы ЛОВ магнитное поле было однородным, а его максимальное значение достигало $30 \mathrm{kOe}$ К Кромка катода находилась в слабосходящемся магнитном поле, обеспечивающем небольшую компрессию электронного пучка. Диаметр эмиссионной кромки катода был равен $\sim 34 \mathrm{~mm}$, наружный диаметр электронного пучка в центральной части SWS составлял $\sim 26 \mathrm{~mm}$, толщина $\sim 0.4 \mathrm{~mm}$. Конусообразный дифракционный выход (MW out), совмещенный с коллектором электронов $(\mathrm{Col})$, был выполнен в отдельной секции, позволяющей проводить ее замену. Вывод микроволнового излучения в атмосферу осуществлялся 

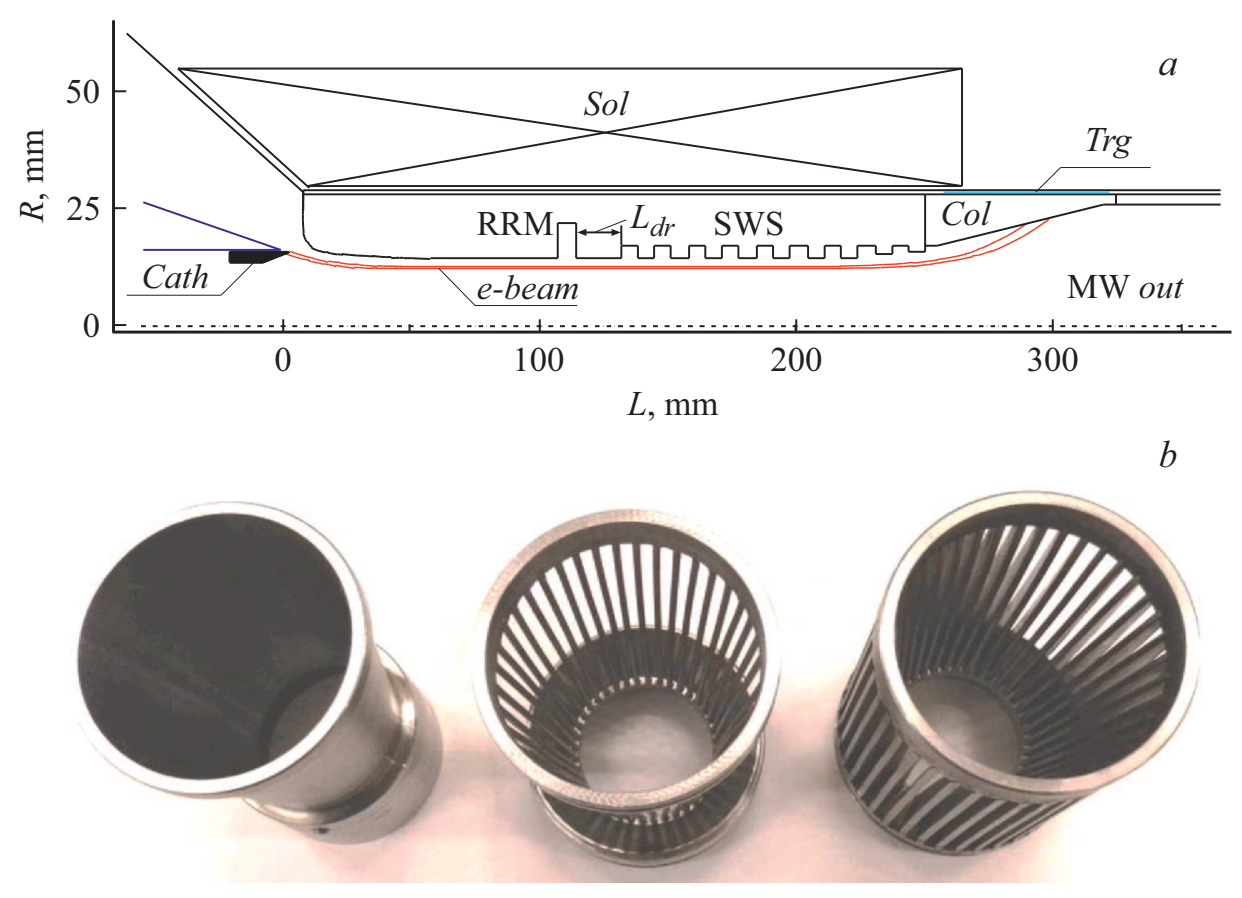

Рис. 1. $a-$ конструктивная схема ЛОВ; $b-$ внешний вид дифракционных выходов ЛОВ: слева - сплошной, в центре - проволочный, справа - пластинчатый.

после преобразования с помощью конвертора рабочей моды $\mathrm{TM}_{01}$ в моду $\mathrm{TE}_{11}$ и последующего формирования гауссова пучка с помощью конического рупора.

В процессе работы использовались три типа дифракционных выходов (коллекторов), выполненных из немагнитной нержавеющей стали: сплошной, пластинчатый и проволочный. Размеры всех дифракционных выходов были идентичными: входной внутренний диаметр равен $32 \mathrm{~mm}$, выходной внутренний диаметр $52 \mathrm{~mm}$, длина $74 \mathrm{~mm}$. Проволочный выход был выполнен из равномерно расположенных по окружности 40 спиц диаметром $1.5 \mathrm{~mm}$, пластинчатый - из такого же числа равномерно расположенных пластин толщиной $1.5 \mathrm{~mm}$, внутренние ребра которых имели округлую форму, подобную форме спиц. Концевые крепления пластин и проволок были осуществлены путем их плавного сопряжения с переходными цилиндрами длиной $\sim 7 \mathrm{~mm}$, также изготовленными из немагнитной нержавеющей стали. Осаждение электронного пучка происходило на диаметре коллекторов $\sim 45-46 \mathrm{~mm}$, где отношение площади щелей к площади поверхностей проволочного и пластинчатого коллекторов составляло $\sim 0.6$. Внешний вид коллекторов приведен на рис. $1, b$.

В ходе экспериментов с помощью осциллографов регистрировались форма и величина наносекундных импульсов ускоряющего напряжения (измеряемого с помощью емкостного делителя, установленного в области катододержателя), тока электронного пучка (измеряемого с помощью установленного там же пояса Роговского) и микроволнового излучения (измеряемого детектором на горячих электронах, установленным на расстоянии $\sim 5 \mathrm{~m}$ от фазового центра рупора). Кроме того, регистрировались колоколообразные $18 \mathrm{~ms}$ импульсы тока питания соленоида, измеряемые с помощью шунта, установленного в цепи питания соленоида.

Структура электронного пучка и его поперечные размеры в рабочем пространстве ЛОВ контролировались по следам на капролоновых мишенях, а в области коллектора - по следам на мишенях $(\operatorname{Trg})$ из винипросовой пленки толщиной $0.2 \mathrm{~mm}$.

Поскольку на выходе ЛОВ с дифракционным выходом могут присутствовать высшие гармоники, способные вносить значительные искажения в показания используемого СВЧ-детектора на горячих электронах даже при относительно небольшой доле их мощности [7], перед основными измерениями их проявления были минимизированы путем изменения сдвига фаз между основной и второй гармоникой за счет изменения длины приемного волноводного тракта детектора.

Импульсы микроволнового излучения для всех вариантов дифракционного выхода регистрировались СВЧ-детектором начиная с $H_{0}=18 \mathrm{kOe}$, при этом мощность импульсов нарастала по мере его увеличения до $H_{0}=30 \mathrm{kOe}$. Длительность импульсов микроволнового излучения для всех вариантов дифракционного выхода находилась в пределах $6-8 \mathrm{~ns}$ и изменялась незначительно при смене дифракционного выхода и величины ведущего магнитного поля. Зависимости относительной мощности и длительности импульсов микроволнового излучения от величины ведущего магнитного поля при- 
ведены на рис. 2, a. Длительность импульсов измерялась по уровню 0.5 .

Характерные осциллограммы ускоряющего напряжения, тока электронного пучка и показаний микроволнового детектора при напряженности ведущего магнитного поля $H_{0}=28 \mathrm{kOe}$ для сплошного, пластинчатого и проволочного дифракционных выходов приведены на рис. $2, b$.

Следы электронного пучка на винипросовых мишенях, устанавливаемых за пластинчатым и проволочным коллекторами, позволяющие судить об их прозрачности для электронного пучка, приведены на рис. 3 .

Оценка полной мощности выходного излучения ЛОВ, выполненная по формуле $P=p_{\max } S_{e}$ для всех вариантов дифракционных выходов при $H_{0}=28 \mathrm{kOe}$, составила $\sim 0.9 \mathrm{GW}$. Здесь $p_{\max }\left[\mathrm{W} / \mathrm{cm}^{2}\right]$ - плотность потока энергии микроволнового излучения в центре выходного гауссова пучка, $S_{e}\left[\mathrm{~cm}^{2}\right]$ - площадь поперечного сечения гауссова пучка на уровне удельной мощности $p_{\max } / e$.

Проведенные эксперименты показали, что замена сплошного дифракционного выхода на его пластинчатый и проволочный аналоги незначительно влияет на форму импульса выходного микроволнового излучения и не уменьшает его мощности. Опасения по поводу того,
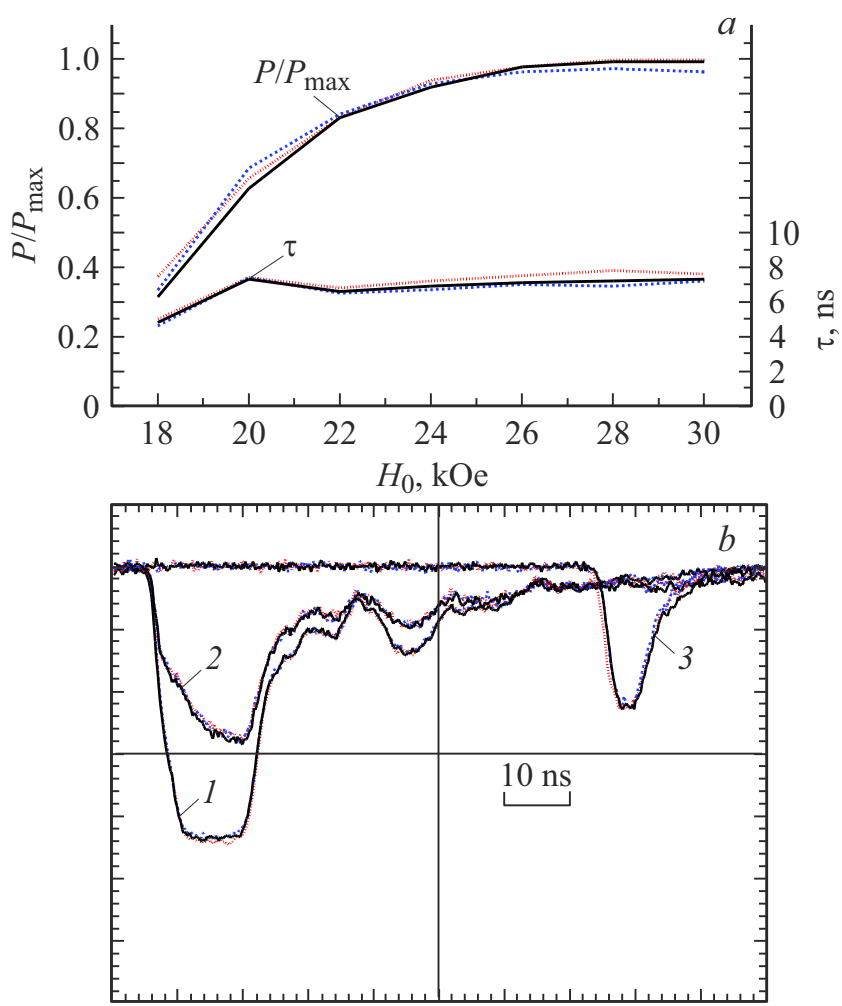

Рис. 2. $a-$ зависимости относительной мощности $\left(P / P_{\max }\right)$ и длительности импульсов микроволнового излучения $(\tau)$ от величины ведущего магнитного поля; $b-$ осциллограммы импульсов: 1 - ускоряющего напряжения, 2 - тока пучка, 3 - микроволнового излучения. Сплошная линия соответствует сплошному коллектору, штриховая - пластинчатому коллектору, пунктирная - проволочному коллектору.

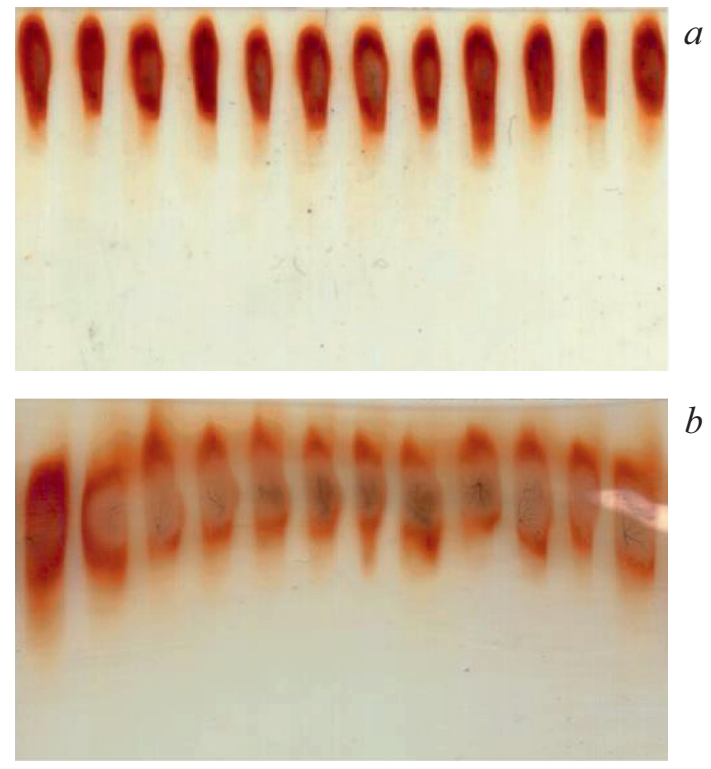

Рис. 3. Следы электронного пучка. $a-$ пластинчатый коллектор, $b-$ проволочный коллектор.

что от продольно-щелевых дифракционных выходов возникнут отражения, оказывающие негативное влияние на работу ЛОВ и вывод микроволнового излучения, не подтвердились.

Изучение следов на мишенях позволяет сделать вывод, что проволочный коллектор обладает существенно большей прозрачностью по сравнению с пластинчатым, что, вероятно, связано с осаждением значительной части электронов на гранях пластинчатого выхода.

Переход от сплошного коллектора к продольнощелевому позволяет уменьшить объем и локализацию в области дифракционного выхода коллекторной плазмы, десорбированных газов, паров и микрочастиц, образующихся на поверхности коллектора под действием электронного пучка, уменьшить поверхностные отражения микрочастиц, улучшить их адсорбцию и удаление.

К достоинствам продольно-щелевых дифракционных выходов также следует отнести их фильтрующие свойства (подавление ТЕ-волн в выходном излучении).

\section{Конфликт интересов}

Авторы заявляют, что у них нет конфликта интересов.

\section{Список литературы}

[1] Н.Ф. Ковалев, М.И. Петелин, М.Д. Райзер, А.В. Сморгонский, Л.Э. Цопп, Письма в ЖЭТФ, 18 (4), 232 (1973). [N.F. Kovalev, M.I. Petelin, M.D. Raizer, A.V. Smorgonski, L.E. Tsopp, JETP Lett., 18 (4), 138 (1973). http://jetpletters.ru/ps/1562/article_23909.pdf].

[2] V.V. Rostov, M.I. Yalandin, G.A. Mesyats, IEEE Trans. Plasma Sci., 36 (3), 655 (2008). DOI: 10.1109/TPS.2008.923736 
[3] J. Zhang, D. Zhang, Y. Fan, J. He, X. Ge, X. Zhang, J. Ju, T. Xun, Phys. Plasmas, 27 (1), 010501 (2020). DOI: $10.1063 / 1.5126271$

[4] С.Е. Андреев, И.Л. Богданкевич, Н.Г. Гусейн-заде, О.Т. Лоза, Физика плазмы, 47 (3), 224 (2021). DOI: 10.31857/S0367292121030021 [S.E. Andreev, I.L. Bogdankevich, N.G. Gusein-zade, O.T. Loza, Plasma Phys. Rep., 47 (3), 257 (2021). DOI: 10.1134/S1063780X21030028].

[5] М.Б. Гойхман, А.В. Громов, В.В. Кладухин, Н.Ф. Ковалев, Н.Г. Колганов, А.В. Палицын, С.П. Храмцов, Письма в ЖТФ, 37 (7), 87 (2011). [M.B. Goykhman, A.V. Gromov, V.V. Kladukhin, N.F. Kovalev, N.G. Kolganov, A.V. Palitsin, S.P. Khramtsov, Tech. Phys. Lett., 37 (4), 333 (2011). DOI: $10.1134 / \mathrm{S} 1063785011040109]$.

[6] С.Д. Коровин, В.В. Ростов, Е.М. Тотьменинов, Письма в ЖТФ, 31 (10), 17 (2005). [S.D. Korovin, V.V. Rostov, E.M. Tot'meninov, Tech. Phys. Lett., 31 (5), 411 (2005). DOI: $10.1134 / 1.1931784]$.

[7] М.Б. Гойхман, Н.Ф. Ковалев, Н.Г. Колганов, А.В. Палицын, Письма в ЖТФ, 30 (12), 28 (2004). [M.B. Goykhman, N.F. Kovalev, N.G. Kolganov, A.V. Palitsin, Tech. Phys. Lett., 30 (6), 500 (2004). DOI: 10.1134/1.1773349]. 